\title{
Genomic analysis of halophilic bacterium, Lentibacillus sp. CBA3610, derived from human feces
}

Seung Woo Ahn' ${ }^{1}$, Se Hee Lee ${ }^{1}$, Hong-Seok Son², Seong Woon Roh ${ }^{1 *}$ (D) and Yoon-E Choi ${ }^{3^{*}}$

\begin{abstract}
Background: Lentibacillus species are gram variable aerobic bacteria that live primarily in halophilic environments. Previous reports have shown that bacteria belonging to this species are primarily isolated from salty environments or food. We isolated a bacterial strain CBA3610, identified as a novel species of the genus Lentibacillus, from a human fecal sample. In this report, the whole genome sequence of Lentibacillus sp. CBA3610 is presented, and genomic analyses are performed.

Results: Complete genome sequence of strain CBA3610 was obtained through PacBio RSII and Illumina HiSeq platforms. The size of genome is 4,035,571 bp and genes estimated to be 4714 coding DNA sequences and 64 tRNA and 17 rRNA were identified. The phylogenetic analysis confirmed that it belongs to the genus Lentibacillus. In addition, there were genes related to antibiotic resistance and virulence, and genes predicted as CRISPR and prophage were also identified. Genes related to osmotic stress were found according to the characteristics of halophilic bacterium. Genomic differences from other Lentibacillus species were also confirmed through comparative genomic analysis.

Conclusions: Strain CBA3610 is predicted to be a novel candidate species of Lentibacillus through phylogenetic analysis and comparative genomic analysis with other species in the same genus. This strain has antibiotic resistance gene and pathogenic genes. In future, the information derived from the results of several genomic analyses of this strain is thought to be helpful in identifying the relationship between halophilic bacteria and human gut microbiota.
\end{abstract}

Keywords: Lentibacillus sp. CBA3610, Complete genome sequence, Gut microbiota, Halophile

\section{Background}

Lentibacillus is a gram-variable, aerobic or facultatively anaerobic, and halophilic bacterial genus of the family Bacillaceae in the phylum Firmicutes [1]. This genus has been classified as a new genus and species, different from the genus Virgibacillus, Salibacillus, Gracilibacillus, and Halobacillus, which was identified to have

*Correspondence: seong18@gmail.com; yechoi@korea.ac.kr

${ }^{1}$ Microbiology and Functionality Research Group, World Institute of Kimchi, Gwangju 61755, Republic of Korea

${ }^{3}$ Division of Environmental Science and Ecological Engineering, Korea University, Seoul 02841, Republic of Korea

Full list of author information is available at the end of the article close phylogenetic relationship, based on 16S rRNA gene sequence analysis and phenotypic characteristics, such as unique lipid content and fatty acid profile [2]. The presence of halophilic prokaryotes in the human gut has been confirmed by various molecular biological and next-generation sequencing (NGS) techniques. However, little had been known about the information of halophilic microorganisms inhabiting the human gut [3]. Recently, halophilic microorganisms have been isolated and reported through development of culturomics $[4,5]$. The previous study suggested that the presence of halophilic microbiota in the gut is associated with high salinity in the gut. High salinity of human gut changes the halophilic microbiota which could be related to human diseases such as 
obesity [5]. Therefore, further studies of halophilic bacteria isolated from the human gut could be helpful in elucidating the relationship between halophilic bacteria and human health. We isolated a bacterium belonging to the Lentibacillus species from human fecal sample, identified its whole genome sequence through NGS, and analyzed information on the genes that could have a pathogenic effect on humans. In addition, we performed phylogenetic analysis based on 16S rRNA gene sequence and comparative genomic analysis with other species of genus Lentibacillus.

\section{Methods}

\section{Bacterial strain isolation}

Strain CBA3610 was isolated from a stool sample from a 28-year-old healthy male in Gwangju, Republic of Korea. The fecal sample was enriched in Deutsche Sammlung von Mikroorganismen und Zellkulturen (DSMZ) medium 372 broth under aerobic conditions at $37{ }^{\circ} \mathrm{C}$ for 7 days, after which $100 \mathrm{~mL}$ of the enriched broth was spread on DSMZ medium 372 agar plates to isolate bacterial strains under aerobic conditions at $37^{\circ} \mathrm{C}$ for $24 \mathrm{~h}$. Strain CBA3610 was isolated from several colonies, and subculturing was performed under the same conditions at least three times.

\section{Genome sequencing, assembly, and gene annotation}

The genomic DNA of the isolated strain was extracted and purified using the MG genomic DNA purification kit (MGmed, Seoul, Korea). The whole genome sequencing was performed using Pacific Biosciences RS II (Pacific Biosciences, Menlo Park, CA) and Illumina HiSeq X Ten (Illumina, San Diego, CA). Each library used for sequencing was constructed using a 20-kb SMRTbell template preparation kit and a TruSeq Nano DNA High Throughput Library kit. The genome was assembled using the protocol of Unicycler ver. 0.4.6 with PacBio SMRT analysis ver. 2.3 [6] and Pilon ver. 1.21 with Illumina HiSeq for error correction [7]. The subread filtering of the PacBio sequences was performed based on the following criteria: minimum subread length 50 , minimum polymerase read quality 75 , and minimum polymerase read length 50 . Adapter/primer contamination of HiSeq raw sequences was confirmed using FastQC (v0.11.9). The genome was annotated using the Pathosystems Resource Integration Center (PATRIC; https://www.patricbrc.org/) ver. 3.6.7, the bacterial bioinformatics database and analysis resource [8]. We constructed a phylogenetic tree based on 16S rRNA gene sequences. To construct the phylogenetic tree, the sequences of $16 \mathrm{~S}$ rRNA gene of strain CBA3610 and related species were aligned using Clustal W [9]. Phylogenetic trees were constructed using MEGA 7, based on the neighbor-joining (NJ) [10], maximum parsimony (MP) [11], and maximum likelihood (ML) [12] algorithms using 1000 bootstrap value [13]. Functional genes were predicted and annotated using Rapid Annotation using Subsystem Technology (RAST; https:// rast.nmpdr.org/) [14]. PathogenFinder (https://cge.cbs. $\mathrm{dtu} . \mathrm{dk} /$ services/PathogenFinder/) was used for predicting pathogenicity towards humans [15]. The presence of Clustered Regularly Interspaced Short Palindromic Repeats (CRISPRs) was detected using the CRISPRfinder server (https://crispr.i2bc.paris-saclay.fr/Server/) [16]. Prophages were confirmed using the PHASTER database (https://phaster.ca/), a phage search tool [17].

\section{Comparative genomics analysis}

Comparative genome analysis was performed using 12 reference strains belonging to the genus Lentibacillus along with strain CBA3610. The genome and amino acid sequences of 12 reference strains are available in the GenBank of National Center for Biotechnology Information (NCBI, Accessed 22 September 2020). The list of strains used in the analysis is summarized in Additional file 1: Table S1. Pan-genome analysis was performed using Bacterial Pan Genome Analysis tool (BPGA). The 50\% sequence identity cut-off was applied to obtain the core genomes of a total of 13 strains using USEARCH (ver. 9.0) [18]. The core genome tree was constructed with the aligned amino acid sequences of common genes of 13 strains using MAFFT (ver. 7.471) [19] and the MEGA 7 with NJ algorithm $[10,13]$. The OrthoANI value was calculated using the Orthologous Average Nucleotide Identity Tool (OAT) provided by EzBioCloud database [20].

\section{Quality assurance}

Before genomic DNA extraction, the single colony of strain CBA3610 was transferred three times in DSMZ medium 372 to obtain pure single colony. After obtaining the whole genome sequence of strain CBA3610, the sequence of the $16 \mathrm{~S}$ rRNA gene, extracted using RNAmmer 1.21 server, was confirmed through the EzBioCloud database.

\section{Results and discussion}

\section{Genome characteristics and annotation data}

After the PacBio subreads filtering process, the total number of bases was 1,186,149,844 and the number of reads was 111,990 . After the HiSeq raw data filtering process, the total number of bases in the filtered dataset was $796,687,476$ and number of reads was 5,276,076. In the de novo assembly process, long-reads of PacBio were assembled using the default option. After de novo assembly with PacBio subreads and error correction using HiSeq reads, the complete genome of Lentibacillus sp. CBA3610 consists of one chromosome (Total length: 4,035,571 bp). 
No plasmid was identified. Chromosome was circular with $42 \% \mathrm{G}+\mathrm{C}$ content. According to the PATRIC annotation results, the genome has 4714 predicted genes, 166 repeat regions, 64 tRNA genes, and 17 rRNA genes. The genome of Lentibacillus sp. CBA3610 was annotated as having one virulence factor, four transporters, four drug targets, and 37 antibiotic resistance genes. The circular map of the genome is shown in Fig. 1, and detailed genomic characteristics are listed in Table 1. A phylogenetic tree was constructed, based on the 16S rRNA gene sequences of the strains with close similarity to the Lentibacillus sp. CBA3610 (Fig. 2A). The similarity of the $16 \mathrm{~S}$ rRNA gene sequence of strain CBA3610 with Lentibacillus salicampi $\mathrm{SF}-20^{\mathrm{T}}$, Lentibacillus salarius $\mathrm{BH} 139^{\mathrm{T}}$ and Lentibacillus halodurans $8-1^{\mathrm{T}}$ was $95.81 \%, 95.63 \%$ and $95.61 \%$, respectively. On the phylogenetic tree based on
Table 1 Complete genome features of Lentibacillus sp. CBA3610

\begin{tabular}{ll}
\hline Property & Value \\
\hline Sequencing platform & PacBio RS II \\
Topology & Circular \\
Genome size (bp) & $4,035,571$ \\
Contig numbers & 1 \\
Genome coverage (fold) & 242 \\
DNA G + C (\%) & 42.04 \\
Number of tRNA genes & 64 \\
Number of rRNA genes & 17 \\
Number of CDSs & 4714 \\
CRISPR candidates & 5 \\
\hline
\end{tabular}

the 16S rRNA gene sequences, strain CBA3610 clustered with Lentibacillus halodurans $8-1^{\mathrm{T}}$ and Lentibacillus salarius $\mathrm{BH} 139^{\mathrm{T}}$. Strain CBA3610 can be considered as

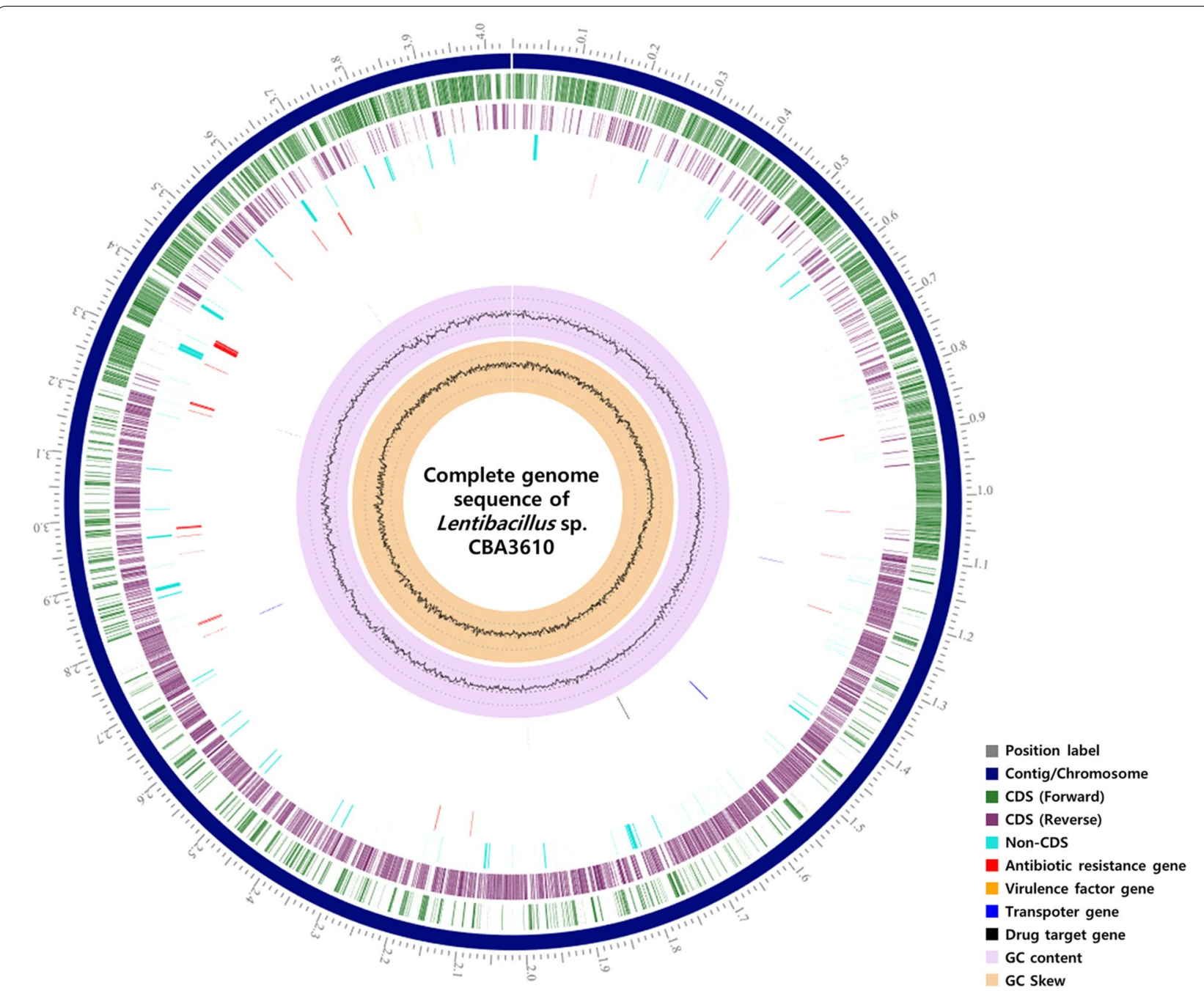

Fig. 1 Circular map of Lentibacillus sp. CBA3610 genome. From outer to inner rings, the individual circles indicate forward CDS, reverse CDS, non-CDS, antibiotic resistance genes, virulence factor gene, transporter gene, drug target gene, GC content, and GC skew 


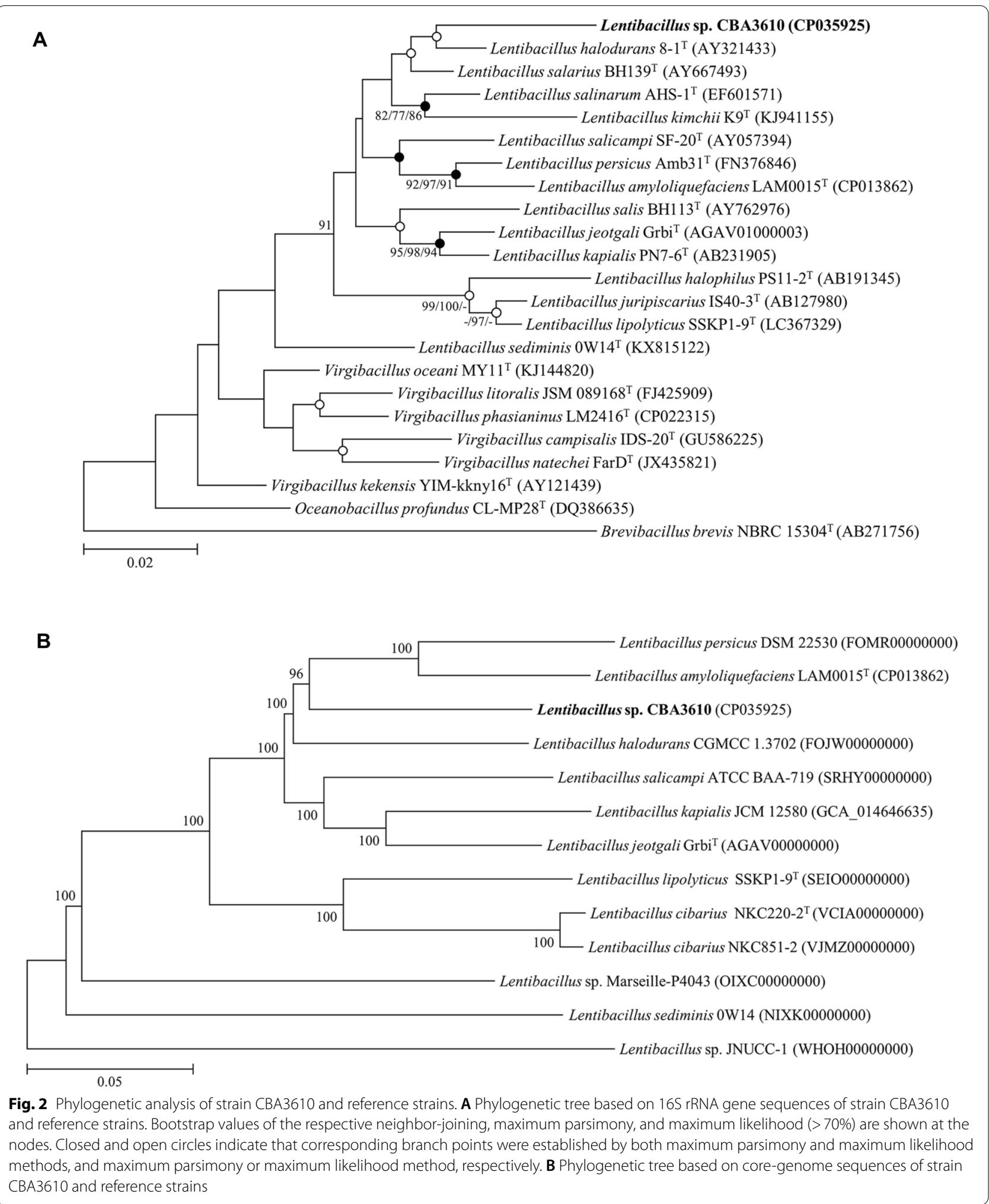


a novel candidate species of the genus Lentibacillus [21]. Based on the results of the RAST annotation, the following categories were classified in the SEED subsystem: amino acids and derivatives (346), carbohydrates (265), protein metabolism (194), cofactors, vitamins, prosthetic groups, and pigments (106) (Additional file 1: Figure S1). Among the 27 categories based on RAST annotation, 55 coding sequences (CDSs) existed in the 'Virulence, Disease and Defense'. Among these, five CDSs were found to belong to the 'Resistance to fluoroquinolones' category related to antibiotic resistance. Based on the results of the PathogenFinder, this strain was not classified as a human pathogen because only one sequence classified as pathogenic, and 14 other sequences classified as non-pathogenic, were identified (Additional file 1: Table S2). The sequence classified as that belonging to the pathogenic family showed $84.78 \%$ similarity to those annotated with the function of $30 \mathrm{~S}$ ribosomal protein S19 in the genome of Listeria monocytogenes 08-5578. CRISPRFinder detected five sequences presumed to be CRISPR candidates (Additional file 1: Table S3), and two incomplete prophage regions were found using PHASTER (Additional file 1: Table S4). Among the incomplete prophage regions, region 1 was confirmed to match PHAGE_Bacill_G_NC_023719 and region 2 matched to PHAGE_Brevib_Jimmer1_NC_029104.

\section{Osmotic stress-related genes}

Taking into consideration of the characteristics of Lentibacillus of being a halophilic bacterium that survives in a high salinity environment, genes related to osmotic stress of strain CBA3610 were analyzed. In the SEED subsystem of the RAST server, total 34 genes classified as related to 'osmotic stress', were identified. Among these, one gene was classified as related to osmoregulation and the remaining 33 genes were annotated to be related to choline and betaine uptake and betaine biosynthesis. The gene involved in osmoregulation encoded the aquaporin family protein, a transporter of glycerol across the cytoplasmic membrane that has limited permeability to small uncharged compounds, such as water. The remaining 33 genes are described as follows. As genes involved in the biosynthesis of osmoprotectant glycine betaine, there is one bet $A$ gene that encodes oxygen-dependent choline dehydrogenase, which converts choline to betaine aldehyde, and three betB genes that encode NAD/NADPdependent betaine aldehyde dehydrogenase, which converts betaine aldehyde to glycine betaine. In addition, there are seven opuD genes that encode glycine betaine transporter, which are involved in glycine betaine uptake, and 12 genes belonging to the opuA gene family (including the opuAA, ориAB, and opuAC gene) that encode glycine betaine/carnitine/choline $\mathrm{ABC}$ transporter. Lastly, there are two ProV genes encoding glycine betaine/ proline betaine transport system ATP-binding protein involved in glycine betaine and proline betaine uptake, four genes belonging to the $o p u B$ gene cluster, including $о p u B A, o p u B B, o p u B C$, and $о p u B D$ genes, encoding glycine betaine/carnitine/choline $A B C$ transporter, one opu $C B$ gene encoding carnitine transport permease protein, and three $\operatorname{sox} A$ genes that encode sarcosine oxidase alpha subunit that converts sarcosine to glycine [22-24].

\section{Comparative genomics}

Results of the pan-genome analysis using BPGA showed that between strain CBA3610 and 12 reference strains, 11,961 genes of pan-genome and 849 genes of core genome were found (Additional file 1: Figure S2). Strain CBA3610 had 2212 accessory genes (present in genome of 2-12 strains) of strain CBA3610 and 449 unique genes present only in genome (Additional file 1: Table S5). In the phylogenetic tree based on the core genome, strain CBA3610 was located close to Lentibacillus persicus, Lentibacillus amyloliquefaciens, and Lentibacillus halodurans; it was confirmed that strain CBA3610 belongs to the genus Lentibacillus (Fig. 2B). The calculated OrthoANI values between strain CBA3610 and the remaining 12 reference strains are summarized in Additional file 1: Table S6. The range of OrthoANI values between strain CBA3610 and the remaining 12 reference strains was $68.69-79.68 \%$, showing the minimum value with strain Lentibacillus sp. JNUCC-1 and the maximum value with strain Lentibacillus halodurans CGMCC 1.3702. This result also supplements that of the phylogenetic analysis described above.

\section{Conclusion}

The sequencing process to obtain the genome of Lentibacillus sp. CBA3610 and general characteristics of the genome were summarized, and additional genomic characteristics were analyzed using various databases. It is predicted that the probability of strain CBA3610 having a pathogenic effect on humans is low. However, considering the ongoing studies to elucidate the relationship between the human gut microbiome and halophilic microbiota, we believe that this genome information may be helpful in future studies.

\section{Supplementary Information}

The online version contains supplementary material available at https://doi. org/10.1186/s13099-021-00436-2.

Additional file 1: Figure S1. Subsystem distribution of Lentibacillus sp. CBA3610 genome using SEED analysis. Figure S2. Pan- and core-genome box plots of Lentibacillus sp. CBA3610 and 12 reference Lentibacillus strains with standard deviations. Table S1. List of strains used in pan-genomic 
analysis. Table S2. PathogenFinder results of Lentibacillus sp. CBA3610. Table S3. CRISPR candidate sequences of Lentibacillus sp. CBA3610. Table S4. Prophages of Lentibacillus sp. CBA3610. Table S5. The numbers of core-, accessory-, and unique genes of Lentibacillus sp. CBA3610 and 12 reference strains. Table S6. OrthoANI values between strain CBA3610 and 12 reference Lentibacillus strains.

\section{Acknowledgements}

\section{Not applicable.}

\section{Authors' contributions}

SWA, SHL, H-SS, and SWR contributed to performing genome analysis and writing manuscript. SWR and YEC contributed to the conception and design of the study. All authors read and approved the final manuscript.

\section{Funding}

This research was supported by a Grant from the World Institute of Kimchi (KE2101-2) funded by the Ministry of Science and ICT; the National Research Foundation of Korea (NRF) Grant funded by the Korea government (MSIP) (2018M3A9F3055925 and 2019R1A2C2087449), Republic of Korea.

\section{Availability of data and materials}

The complete genome data of Lentibacillus sp. CBA3610 has been deposited in DDBJ/EMBL/GenBank, with Accession Number CP035925.

\section{Declarations}

\section{Ethics approval and consent to participate}

The study protocol was approved by the Institutional Review Board (IRB) of Dongshin University (IRB No. DSMOH19-1) and was compliant with all relevant ethical regulations

\section{Consent for publication}

Not applicable.

\section{Competing interests}

The authors declare that they have no competing interests.

\section{Author details}

${ }^{1}$ Microbiology and Functionality Research Group, World Institute of Kimchi, Gwangju 61755, Republic of Korea. ${ }^{2}$ Department of Food Bioscience and Technology, Korea University, Seoul 02841, Republic of Korea. ${ }^{3}$ Division of Environmental Science and Ecological Engineering, Korea University, Seoul 02841, Republic of Korea.

Received: 4 December 2020 Accepted: 10 June 2021 Published online: 23 June 2021

\section{References}

1. Jung MJ, Roh SW, Kim MS, Bae JW. Lentibacillus jeotgali sp. nov., a halophilic bacterium isolated from traditional Korean fermented seafood. Int J Syst Evol Microbiol. 2010;60:1017-22.

2. Yoon JH, Kang KH, Park YH. Lentibacillus salicampi gen. nov., sp. nov., a moderately halophilic bacterium isolated from a salt field in Korea. Int J Syst Evol Microbiol. 2002;52:2043-8.

3. Seck EH, Dufour JC, Raoult D, Lagier JC. Halophilic \& halotolerant prokaryotes in humans. Future Microbiol. 2018;13:799-812

4. Lagier JC, Khelaifia S, Alou MT, Ndongo S, Dione N, Hugon P, et al. Culture of previously uncultured members of the human gut microbiota by culturomics. Nat Microbiol. 2016;1:16203.
5. Seck EH, Senghor B, Merhej V, Bachar D, Cadoret F, Robert C, et al. Salt in stools is associated with obesity, gut halophilic microbiota and Akkermansia muciniphila depletion in humans. Int J Obes. 2019;43(4):862-71.

6. Wick RR, Judd LM, Gorrie CL, Holt KE. Unicycler: resolving bacterial genome assemblies from short and long sequencing reads. PLoS Comput Biol. 2017;13(6):e1005595.

7. Walker BJ, Abeel T, Shea T, Priest M, Abouelliel A, Sakthikumar S, et al. Pilon: an integrated tool for comprehensive microbial variant detection and genome assembly improvement. PLoS ONE. 2014;9(11):e112963.

8. Wattam AR, Davis JJ, Assaf R, Boisvert S, Brettin T, Bun C, et al. Improvements to PATRIC, the all-bacterial bioinformatics database and analysis resource center. Nucleic Acids Res. 2017:45:D535-42.

9. Thompson JD, Higgins DG, Gibson TJ. CLUSTAL W: improving the sensitivity of progressive multiple sequence alignment through sequence weighting, position-specific gap penalties and weight matrix choice. Nucleic Acids Res. 1994;22(22):4673-80.

10. Saitou N, Nei M. The neighbor-joining method: a new method for reconstructing phylogenetic trees. Mol Biol Evol. 1987:4(4):406-25.

11. Farris JS. Methods for computing wagner trees. Syst Biol. 1970;19(1):83-92.

12. Kluge AG, Farris JS. Quantitative phyletics and the evolution of anurans. Syst Biol. 1969;18(1):1-32.

13. Kumar S, Stecher G, Tamura K. MEGA7: molecular evolutionary genetics analysis version 7.0 for bigger datasets. Mol Biol Evol. 2016;33(7):1870-4

14. Overbeek R, Olson R, Pusch GD, Olsen GJ, Davis JJ, Disz T, et al. The SEED and the rapid annotation of microbial genomes using subsystems technology (RAST). Nucleic Acids Res. 2014:42:D206-14.

15. Cosentino S, Voldby Larsen M, Moller Aarestrup F, Lund O. PathogenFinder - distinguishing friend from foe using bacterial whole genome sequence data. PLOS ONE. 2013;8(10):e77302.

16. Grissa I, Vergnaud G, Pourcel C. CRISPRFinder: a web tool to identify clustered regularly interspaced short palindromic repeats. Nucleic Acids Res. 2007;35:W52-7.

17. Arndt D, Grant JR, Marcu A, Sajed T, Pon A, Liang Y, Wishart DS. PHASTER: a better, faster version of the PHAST phage search tool. Nucleic Acids Res. 2016:44:W16-21.

18. Chaudhari NM, Gupta VK, Dutta C. BPGA- an ultra-fast pan-genome analysis pipeline. Sci Rep. 2016;6:24373.

19. Rozewicki J, Li S, Amada KM, Standley DM, Katoh K. MAFFT-DASH: integrated protein sequence and structural alignment. Nucleic Acids Res. 2019;47:W5-10

20. Lee I, Ouk Kim Y, Park SC, Chun J. OrthoANI: an improved algorithm and software for calculating average nucleotide identity. Int J Syst Evol Microbiol. 2016;66(2):1100-3.

21. Stackebrandt E, Goebel BM. Taxonomic note: a place for DNA-DNA reassociation and $16 \mathrm{~S}$ rRNA sequence analysis in the present species definition in bacteriology. Int J Syst Evol Microbiol. 1994;44(4):846-9.

22. Canovas D, Vargas C, Kneip S, Moron MA, Ventosa A, Bremer E, et al. Genes for the synthesis of the osmoprotectant glycine betaine from choline in the moderately halophilic bacterium Halomonas elongata DSM 3043 USA. Microbiology. 2000;146:455-63.

23. Kappes RM, Kempf B, Bremer E. Three transport systems for the osmoprotectant glycine betaine operate in Bacillus subtilis: characterization of OpuD. J Bacteriol. 1996;178(17):5071-9.

24. Wargo MJ. Homeostasis and catabolism of choline and glycine betaine: lessons from Pseudomonas aeruginosa. Appl Environ Microbiol. 2013;79(7):2112-20

\section{Publisher's Note}

Springer Nature remains neutral with regard to jurisdictional claims in published maps and institutional affiliations. 